\title{
Blackwell's Ordering and Public Information *
}

\author{
Colin M. Campbell \\ Department of Economics \\ Rutgers University \\ New Brunswick, New Jersey 08901 \\ Phone: (732) 932-8259 \\ Fax: (732) 932-7416 \\ campbell@econ.rutgers.edu
}

January, 2002

* This paper has benefited greatly from contributions by Don Brown, Richard McLean, Stephen Morris, David Pearce, Herbert Scarf, and seminar participants at Yale University and Washington University. The author is responsible for any errors. 


\begin{abstract}
We characterize a precise comparative static on welfare and the amount of public information in an economy under uncertainty. Results dating to Hirshleifer (1971) have suggested that information can have negative value in such a setting, but counterexamples using competitive equilibrium outcomes have suppressed general results to this effect. We show that under the solution concept of implementable allocations, the negative relationship between more public information in the sense of Blackwell and welfare is fully general. Furthermore, Blackwell's ranking is necessary as well as sufficient to obtain our ranking, and hence ours provides an equivalent characterization of his ordering.

Journal of Economic Literature Classification Number: D80.
\end{abstract}

Keywords: Blackwell's Ordering, Information, Risk Sharing 


\section{INTRODUCTION}

One of the signature topics of information theory is the relationship between the positive, abstract question of what constitutes more information, and the normative, contextual question of what constitutes more valuable information. In certain settings, the notion that more information is valuable is so axiomatic that the answer to the one of these questions is interpreted as necessarily answering the other. This is the case, for instance, for the ordering of information formalized by Blackwell [1]. Blackwell's criterion for ranking different information may be characterized by the condition that any two decision makers, each of whom faces a (distinct) nonstrategic choice under uncertainty, should have the same preferences over which information is better for her particular problem. Equivalent mathematical conditions for this criterion are often invoked as a formalization of what constitutes more information in an economic model.

Two important classes of economic environment have been considered in which more information is possibly not more valuable. One is strategic environments, in which agents incorporate the quality of others' information into their own decisions. Inefficiency created by the presence of adverse selection, for instance, represents a case in which the possession of more accurate information may reduce an agent's welfare. The other class consists of neoclassical economies making state-contingent trades and production choices under uncertainty, in which information about the final state at the time of trade is public and common knowledge. An early paper identifying that information may have a negative value in such a setting is Hirshleifer [6], which provides a variant of the following example. Consider a pure exchange economy in which there is one state-contingent commodity, and consumers are risk-averse von Neumann-Morgenstern expected utility maximizers. Each consumer's utility is strictly increasing in the amount of final good she consumes, so no Pareto-improving ex-post exchange is possible; however, consumers may exchange state-contingent claims before the uncertainty is resolved to their mutual benefit. Now suppose that the consumers have common access to a signal about the final state, which they observe before any exchange takes place. Under the maximum possible information, the final state is fully revealed; however, this precludes mutually beneficial exchange, since all consumers now only value the commodity in the state that is certain 
to be realized. Thus, among all information that the economy might learn about the final state in advance of trade, fully revealing information is the worst possible.

Hirshleifer's intriguing observation has spawned a substantial literature on the relationship between welfare and the amount of public information in an economy under uncertainty. Important contributions include Marshall [8], Green [4] and Hakansson et. al. [5], and, more recently, Eckwert and Zilcha [3] and Schlee [9]. A primary question of interest has been whether Hirshleifer's result, in which the maximum possible amount of information is compared to an arbitrary instance of less information, generalizes to all possible pairs of information structures that might be ordered by their informative content. In addressing this question, the above papers, and the literature in general, has used competitive price equilibrium as the solution concept to attach an outcome to a particular instance of an economy and information. Under this approach, the results have been mixed. Marshall [6] strongly suggests and Green [4] proves formally that when the case of no information is compared to an arbitrary instance of some information, it is impossible for all agents to have higher ex-ante utilities in an equilibrium under the latter; this follows from the logic that, under conditions in which the first welfare theorem holds, any equilibrium under no information is ex-ante Pareto efficient. However, it is not necessarily true that the equilibrium outcome under no information ex-ante Pareto dominates the equilibrium under better information. Furthermore, when two information structures are considered that do not lie at the extremes of uninformative and fully revealing, the equilibrium outcome of either may ex-ante Pareto dominate the other, even if the structures can be ordered by some criterion. Thus, there are no very general results on the ex-ante Pareto ranking of competitive equilibrium outcomes across information structures.

In light of the nonexistence of such results, the literature has focused on conditions under which more information is identifiably better or worse for an economy under uncertainty. Green [4] considers a partial equilibrium model with futures contracts but no state-contingent contracts, and hence incomplete markets, and derives sufficient conditions for an agent to prefer more information in this setting. Eckwert and Zilcha [3] consider dynamic economies with some uninsurable risk and production in which production choices are made under uncertainty about productivity, and show that more 
information may be beneficial by allowing superior savings behavior. Schlee [9] returns to the complete-market exchange case to seek circumstances under which the firm Pareto ranking of Hirshleifer's example generalizes to arbitrary ordered pairs of information structures. He shows that more information always leads to an ex-ante Pareto regression in competitive equilibrium under any of three conditions: there is no aggregate uncertainty across states; there is a set of risk-neutral consumers who can perfectly insure all risk-averse consumers; or the preferences of the consumers are aggregable, in the sense that aggregate demand is independent of the initial distribution of endowments, and hence can be represented by the demand of a single representative consumer. Schlee's results reveal that the kind of relationship between public information and welfare in an economy under uncertainty hinted at by Hirshleifer's example in fact holds under a wide array of interesting circumstances. However, a large set of environments remains outside of those that satisfy Schlee's sufficient conditions.

To deepen the understanding of the relationship between public information and welfare, this paper departs from the previous literature in the use of competitive equilibrium as the solution concept. In place of competitive equilibrium, we use the set-valued solution concept of allocations that are implementable. We define an allocation to be implementable if it is feasible under the economy's resource and production constraints, and if it yields each consumer at least as much expected utility as her endowment given (common) beliefs at the time of exchange. In an Edgeworth box economy, for instance, the set of implementable allocations are those lying in the (lens-shaped if preferences are strictly convex) area between the consumers' indifference curves through the endowment point. The set of implementable allocations represents all allocations that a social planner could choose for the economy under the constraints that the choice must be feasible, and that any consumer who is assigned a bundle giving her less utility than her endowment could instead withdraw from the economy and consume her endowment. We note that any competitive equilibrium allocation lies in this set, though the set is generally well-defined even when a competitive equilibrium does not exist.

Using sets of implementable allocations as the solution concept, we can apply the partial welfare ordering in which set $A$ is ordered ahead of set $B$ if each allocation in $B$ is ex-ante weakly Pareto dominated by some allocation in $A$. Thus, in the social planner 
framework, $A$ is preferred to $B$ if any allocation the planner could choose in $B$ is worse than some allocation the planner could choose in $A$. This allows a comparative static exercise analogous to the one performed on competitive equilibrium allocations.

We derive two main results. The first provides a direct answer to the question of how public information relates to welfare in an economy under uncertainty, using the welfare criterion on sets of allocations laid out above. For any two information structures that can be ordered by Blackwell's criterion, the set of implementable allocations when there is less information dominates the set of implementable allocations when there is more information. So, for instance, fixing the competitive equilibrium allocation when there is more information, even if it is the case that not all consumers are better off ex-ante in the competitive equilibrium allocation when there is less information, there is necessarily some implementable allocation under less information that does make all consumers better off ex-ante. The intuition for why this is so relates directly to a particular way of interpreting Blackwell's theorem. We expand on this later in the paper, but provide a brief intuition here. Signals bear on the set of implementable allocations only via the consumers' participation, or individual rationality, constraints. When information structure $A$ is ordered ahead of information structure $B$ by Blackwell's ordering, we can roughly interpret $A$ as resulting from the observation of some information additional to $B$. Under $A$, individual rationality must be respected for all possible realizations of that additional information. But under $B$, the constraints across realizations of the additional information may be pooled, and hence a larger set of allocations are individually rational. In this setting, more information is a nuisance: it has no instrumental value to the economy, which can make state-contingent decisions, and thus it merely serves to contract the set of interim exchanges that consumers are willing to make.

Our second main result is that (reverse) Blackwell dominance is not only sufficient for the set of allocations implementable under one information structure to dominate the set implementable under another, it is also necessary when one considers a general class of economies. That is, if Blackwell's criterion does not order information structure $A$ ahead of information structure $B$, then it is possible to construct an economy and find an allocation implementable for that economy under $A$ that is not ex-ante Pareto dominated by any allocation implementable under $B$. Thus, our criterion generates a 
partial ordering on information structures that is an exact mirror image of Blackwell's ordering. Employing the definition of Blackwell's criterion using decision problems, we can state our results by saying that information structure $A$ is better than information structure $B$ for all nonstrategic decision-makers facing uncertainty if and only if $B$ is better than $A$ for all economies under uncertainty.

Our focus on implementable allocations as a solution concept rather than competitive equilibrium is not based on a subjective preference for the former. Rather, we wish to highlight the general forces that cause public information to have negative value. As stated above and demonstrated below, under implementable allocations the negative value of public information is unambiguous. We submit that insofar as public information also exhibits this negative value under competitive equilibrium, it is for the same reason: gains to trade are contracted when more information is revealed. That competitive equilibrium need not always preserve the monotonicity of the relationship is in keeping with the idiosyncratic comparative statics that competitive equilibrium is apt to generate, e.g., the example in which a consumer in an exchange economy derives an improved equilibrium utility by disposing of part of her endowment. In the case of the relationship between public information and welfare there is no generally accepted intuition, and hence individual examples showing that more public information can be better or worse under competitive equilibrium may promote a perspective of ambiguity on the subject. Our goal is to show convincingly that the notion that more information harms an economy under uncertainty, as we draw from Hirshleifer's example and Schlee's results, is in fact the natural intuition.

The paper proceeds in four additional sections. The first formalizes the class of economies we consider and defines our partial orderings. The second defines Blackwell's ordering and contains our main results. The third discusses intuition for the results. The fourth concludes.

\section{THE ENVIRONMENT}

There is a finite set $\Omega$ of $M$ states of the world containing elements $\omega$. This set is held fixed throughout the analysis. An economy $E$ is described by the following fundamentals. There is a set of $I$ consumers indexed by $i$. Consumer $i$ is characterized by: a statecontingent endowment $e_{i}(\omega)$, which for each $\omega$ is a point in the consumption set $X$, a 
vector space; and a state-contingent von Neumann-Morgenstern utility function $u_{i}\left(x_{i}, \omega\right)$, concave in $x_{i}$ for every $\omega$, over sure-thing consumption $x_{i}$ in state $\omega$. State-contingent production is possible and is summarized by state-contingent production possibility sets $X(\omega)$. It is assumed that for every $\omega$ the grand endowment $\sum_{i} e_{i}(\omega)$ is in $X(\omega)$, so that null production is possible. This nests the special case of an exchange economy with no disposal, in which $X(\omega)$ is the singleton set $\left\{\sum_{i} e_{i}(\omega)\right\}$ for all $\omega$. Finally, the realized state is generated according to a probability function $f(\omega)$, which is common knowledge among the consumers and satisfies $f(\omega)>0$ for all $\omega$ and $\sum_{\omega \in \Omega} f(\omega)=1$. The set of all economies satisfying these criteria is denoted $\mathcal{E}$.

The economy is presumed to have access to an information structure $\zeta$, comprised of elements $(Y, \Pi) . Y$ is a finite set of $N$ signals with elements $y$; while the set of states $\Omega$ is held fixed, the set of signals $Y$, and its size $N$, may vary across different information structures. $\Pi$ is an $M \times N$ matrix of $M$ probability distributions on the $N$ signals. Specifically, $\pi_{m n}$ is the probability that signal $y_{n}$ is observed given that state $\omega_{m}$ is realized, written $\pi\left(y_{n} \mid \omega_{m}\right)$. Thus, $\Pi$ contains only nonnegative elements, and its rows each sum to 1 ; we refer to the class of matrices having these characteristics as Markov matrices. The elements of $\zeta$ are common knowledge among the consumers. Consumers are assumed to observe, before any economic activity takes place, a single realization of a signal generated from the distribution on states $f(\cdot)$ combined with the conditional distribution $\pi$, i.e., the probability that they observe signal $y$ is $\sum_{\omega \in \Omega} f(\omega) \pi(y \mid \omega)$.

Given an economy $E$ and an information structure $\zeta$, an allocation for the economy is a profile of state- and signal-dependent consumptions $\left(x_{i}(y, \omega)\right)_{i=1}^{I}$. An allocation is defined as feasible if $\sum_{i} x_{i}(y, \omega) \in X(\omega)$ for all $y \in Y$ and all $\omega \in \Omega$. An allocation is defined as individually rational if

$$
\sum_{\omega \in \Omega} f(\omega) \pi(y \mid \omega)\left(u_{i}\left(x_{i}(y, \omega), \omega\right)-u_{i}\left(e_{i}(\omega), \omega\right)\right) \geq 0
$$

for all $y \in Y$ and all $i$. Feasibility simply requires that aggregate consumption lie in the production possibility set for all states. Individual rationality requires that for any signal $y$ that might be realized, each consumer earns a higher expected utility from the allocation conditional on $y$ than she does from consuming her random endowment. This definition of individual rationality captures the feature that all consumers observe the realization 
of the signal before they engage in any economic activity. Thus, when a given consumer determines whether she prefers not to participate in the economy and instead consume her endowment, she uses her posterior beliefs about the state of the world conditional on the realized signal. Any allocation that is both feasible and individually rational given $E$ and $\zeta$ is defined to be implementable. We denote the set of all implementable allocations given $E$ and $\zeta$ by $X^{*}(E, \zeta) \cdot X^{*}(E, \zeta)$ must contain the initial endowment, and hence is nonempty.

We also define implementability for finite lotteries over allocations. Given an economy $E$ and an information structure $\zeta$, a finite lottery is a finite set of $J$ noncontingent allocations $X_{\Delta}$, with typical element $\left(x_{i j}\right)_{i=1}^{I}$, and a signal- and state-dependent probability function $\delta(x \mid y, \omega)$ on $X_{\Delta}$, with $\delta_{j}(y, \omega)$ the probability that allocation $j$ obtains given that signal $y$ and state $\omega$ are realized. Note that any deterministic allocation $\left(x_{i}(y, \omega)\right)_{i=1}^{I}$ can be represented as a finite lottery, with $X_{\Delta}$ the set of all allocations $\left(x_{i}\right)_{i=1}^{I}$ such that $\left(x_{i}\right)_{i=1}^{I}=\left(x_{i}(y, \omega)\right)_{i=1}^{I}$ for some $y \in Y$ and $\omega \in \Omega$, and $\delta_{j}(y, \omega)=1$ if $x_{i j}=x_{i}(y, \omega)$ for all $i, \delta_{j}(y, \omega)=0$ otherwise. A finite lottery is feasible if for every $j, y \in Y$ and $\omega \in \Omega$, $\delta_{j}(y, \omega)>0$ implies $\sum_{i} x_{i j} \in X(\omega)$. A finite lottery is individually rational if for every signal $y$ and consumer $i$,

$$
\sum_{\omega \in \Omega} f(\omega) \pi(y \mid \omega) \sum_{j} \delta_{j}(y, \omega)\left(u_{i}\left(x_{i j}, \omega\right)-u_{i}\left(e_{i}(\omega), \omega\right)\right) \geq 0
$$

A finite lottery is implementable if it is feasible and individually rational. The set of all implementable finite lotteries given $E$ and $\zeta$ is denoted $\Delta^{*}(E, \zeta)$, and is nonempty as it contains the degenerate lottery that puts all probability on the initial endowment.

We now define some partial orderings on information structures. We do so using sets of ordered pairs of information structures to summarize the binary relations we have in mind. The first ordering is economy-dependent and denoted $S(E)$. For two information structures $\zeta$ and $\zeta^{\prime}$, we define $\left(\zeta, \zeta^{\prime}\right) \in S(E)$ if and only if for any allocation $\left(x_{i}^{\prime}\left(y^{\prime}, \omega\right)\right)_{i=1}^{I} \in X^{*}\left(E, \zeta^{\prime}\right)$, there exists an allocation $\left(x_{i}(y, \omega)\right)_{i=1}^{I} \in X^{*}(E, \zeta)$ such that

$$
\sum_{\omega \in \Omega} f(\omega) \sum_{y \in Y} \pi(y \mid \omega) u_{i}\left(x_{i}(y, \omega), \omega\right) \geq \sum_{\omega \in \Omega} f(\omega) \sum_{y^{\prime} \in Y^{\prime}} \pi^{\prime}\left(y^{\prime} \mid \omega\right) u_{i}\left(x_{i}^{\prime}\left(y^{\prime}, \omega\right), \omega\right)
$$


for all $i$. In words, $\zeta$ is ordered over $\zeta^{\prime}$ for economy $E$ if any implementable allocation given $E$ and $\zeta^{\prime}$ is ex-ante weakly Pareto dominated ${ }^{1}$ by some implementable allocation given $E$ and $\zeta$. Building on this ordering, we define the economy-independent ordering $S$ by $\left(\zeta, \zeta^{\prime}\right) \in S$ if and only if $\left(\zeta, \zeta^{\prime}\right) \in S(E)$ for all $E \in \mathcal{E}$. $\zeta$ is ordered over $\zeta^{\prime}$ by $S$ if for any economy $E$ in the class that is considered, and any implementable allocation $\left(x_{i}^{\prime}\left(y^{\prime}, \omega\right)\right)_{i=i}^{I}$ given $E$ and $\zeta^{\prime}$, there is an implementable allocation $\left(x_{i}(y, \omega)\right)_{i=i}^{I}$ given $E$ and $\zeta$ that weakly Pareto dominates $\left(x_{i}\left(y^{\prime}, \omega\right)\right)_{i=i}^{I}$.

We define a second pair of orderings for lotteries, one economy-dependent and one not, as follows. The economy-dependent ordering $S^{\Delta}(E)$ is defined by $\left(\zeta, \zeta^{\prime}\right) \in S^{\Delta}(E)$ if and only if for any implementable lottery $\left(X_{\Delta}, \delta^{\prime}\right) \in \Delta^{*}\left(E, \zeta^{\prime}\right)$, there exists an implementable lottery $\left(X_{\Delta}, \delta\right) \in \Delta^{*}(E, \zeta)$ (note that the set $X_{\Delta}$ is the same for each lottery) such that for every state $\omega \in \Omega$ and every allocation $j$ in $X_{\Delta}$,

$$
\sum_{y \in Y} \pi(y \mid \omega) \delta_{j}(y, \omega)=\sum_{y^{\prime} \in Y^{\prime}} \pi\left(y^{\prime} \mid \omega\right) \delta_{j}^{\prime}\left(y^{\prime}, \omega\right)
$$

In words, the probability that allocation $j$ is chosen conditional on any particular state being realized is identical across the two lotteries. Thus, $\zeta$ is ordered over $\zeta^{\prime}$ given $E$ by $S^{\Delta}(E)$ if any ex-ante probability distribution over state-specific allocations that can be induced by a lottery that is implementable given $E$ and $\zeta^{\prime}$ can also be induced by some lottery that is implementable given $E$ and $\zeta$. The economy-independent ordering $S^{\Delta}$ is defined by $\left(\zeta, \zeta^{\prime}\right) \in S^{\Delta}$ if and only if $\left(\zeta, \zeta^{\prime}\right) \in S^{\Delta}(E)$ for all $E \in \mathcal{E}$.

Deterministic allocations (for given $y$ and $\omega$ ), as are found in the set $X^{*}(E, \zeta)$, are possibly more appealing from an implementation perspective than lotteries, in that they do not require a planner to have the ability to randomize over allocations. However, the ordering implied by $S^{\Delta}$ is stronger than that implied by $S$, in that the set of implementable lotteries over allocations is finer than the set of implementable ex-ante expected utility profiles, and thus $S^{\Delta}$ may merit independent consideration. In particular, if $S^{\Delta}$ orders information structure $\zeta$ ahead of information structure $\zeta^{\prime}$, then $S$ does as well:

Proposition 1: $S^{\Delta} \subseteq S$

1 We use weak Pareto dominance to include the case in which the ex-ante expected utility of each consumer is identical across the two allocations. Thus, this ordering is reflexive. 
Proof: Suppose $\left(\zeta, \zeta^{\prime}\right) \in S^{\Delta}$. Fix $E$. Choose an arbitrary deterministic allocation $\left(x_{i}^{\prime}\left(y^{\prime}, \omega\right)\right)_{i=1}^{I} \in X^{*}\left(E, \zeta^{\prime}\right)$. As described in the text, $\left(x_{i}^{\prime}\left(y^{\prime}, \omega\right)\right)_{i=1}^{I}$ has a lottery representation $\left(X_{\Delta}^{\prime}, \delta^{\prime}\right)$. Since $\left(\zeta, \zeta^{\prime}\right) \in S^{\Delta}$, there is a lottery $\left(X_{\Delta}^{\prime}, \delta\right) \in \Delta^{*}(E, \zeta)$ such that for every $j$ and every $\omega$,

$$
\sum_{y \in Y} \pi(y \mid \omega) \delta_{j}(y, \omega)=\sum_{y^{\prime} \in Y^{\prime}} \pi\left(y^{\prime} \mid \omega\right) \delta_{j}^{\prime}\left(y^{\prime}, \omega\right)
$$

Since lottery $\left(X_{\Delta}^{\prime}, \delta\right)$ induces an identical ex-ante distribution over state-specific consumption profiles, it yields all consumers the same ex-ante expected utility under $\zeta$ as does $\left(X_{\Delta}^{\prime}, \delta^{\prime}\right)$ under $\zeta^{\prime}$. Consider now the deterministic allocation $\left(x_{i}(y, \omega)\right)_{i=1}^{I}=$ $\left(\sum_{j} \delta_{j}(y, \omega) x_{i j}\right)_{i=1}^{I}$. This allocation is feasible by convexity of $X(\omega)$ and feasibility of $X_{\Delta}^{\prime}$. Because all consumers are risk averse, they weakly prefer $\left(x_{i}(y, \omega)\right)_{i=1}^{I}$ to lottery $\left(X_{\Delta}^{\prime}, \delta\right)$ for all $(y, \omega)$, so implementability of $\left(X_{\Delta}, \delta\right)$ implies the implementability of $\left(x_{i}(y, \omega)\right)_{i=1}^{I}$, and $\left(x_{i}(y, \omega)\right)_{i=1}^{I}$ weakly Pareto dominates $\left(X_{\Delta}, \delta\right)$. Thus, $\left(x_{i}(y, \omega)\right)_{i=1}^{I}$ weakly Pareto dominates $\left(X_{\Delta}, \delta^{\prime}\right)$ and $\left(x_{i}^{\prime}\left(y^{\prime}, \omega\right)\right)_{i=1}^{I}$.

Later results will establish that the two orderings are in fact equivalent.

\section{BLACKWELL'S CRITERION AND PUBLIC INFORMATION}

We now review Blackwell's criterion for ordering information structures and compare that ordering to those we have defined. As described in the introduction, Blackwell's ordering is often defined in the context of a single decision maker. The decision maker chooses an action $a$ from some set $A$. Her von-Neumann Morgenstern utility function $u(\cdot, \cdot)$ is defined on pairs $(a, \omega) \in A \times \Omega$. She is allowed to condition her action on the realized signal $y$. She prefers information structure $\zeta$ to $\zeta^{\prime}$ if she achieves a higher ex-ante expected utility behaving optimally with respect to signals generated by the former than by the latter. Blackwell's criterion for ordering information structures is that $\zeta$ is ordered over $\zeta^{\prime}$ if and only if a decision maker would prefer $\zeta$ to $\zeta^{\prime}$ for any decision problem, as characterized by the set $A$, utility function $u(\cdot, \cdot)$, and a prior on the distribution over states $f(\omega)$. Equivalently, if randomized actions are allowed, the criterion requires that for any decision problem, any feasible distribution of payoffs under $\zeta^{\prime}$ can be duplicated under $\zeta$. 
Blackwell and others have established that the above criterion is equivalent to numerous conditions on the matrices of conditional signal probabilities $\Pi$ and $\Pi^{\prime}$ (see, e.g., Blackwell [1] for an original exposition, or Blackwell and Girshick [2] or Kihlstrom [7] for summaries). A dual approach is to consider not the probabilities with which signals are generated by states, as in $\Pi$ and $\Pi^{\prime}$, but rather the posterior probabilities over states conditional on signals. When there are $N$ signals, this would be represented by an $N \times M$ Markov matrix $P$, along with a marginal distribution over signals represented by a Markov $N$-vector $g$. Equivalent expressions for Blackwell's criterion have been established where the pair $(P, g)$ is the unit of analysis rather than $(Y, \Pi)$, i.e., there exist equivalent binary relations over pairs $(P, g)$ and $\left(P^{\prime}, g^{\prime}\right)$. Use of Bayes' rule provides the link between the two approaches: any marginal distribution over states $f$ and any distribution of signals conditional on states $\Pi$ imply a unique marginal distribution over signals $g$, given by the product $f \Pi$, and for any signal $y_{n}$ that is observed with positive probability $\left(g_{n}>0\right)$, element $P_{n m}$ of matrix $P$ is $f_{m} \Pi_{m n} / g_{n}$. If $g_{n}=0$ then row $n$ of matrix $P$ may be specified arbitrarily; for convenience it is assumed to equal the marginal distribution $f$, i.e., $P_{n m}=f_{m}$.

For our purposes, it is most convenient and intuitive to use forms of Blackwell's criterion defined on the $(P, g)$ representation of an information structure. Two such equivalent criteria are as follows:

Theorem 1 (Blackwell) : $\left(P^{\prime}, g^{\prime}\right)$ is ordered ahead of $(P, g)$ by Blackwell's criterion if and only if there exists an $N \times N^{\prime}$ Markov matrix $T$ such that $P=T P^{\prime}$ and $g T=g^{\prime}$.

Theorem 2 (Blackwell) : $\left(P^{\prime}, g^{\prime}\right)$ is ordered ahead of $(P, g)$ by Blackwell's criterion if and only if for any real-valued, concave function $\varphi(\cdot)$ defined on $1 \times M$ Markov vectors, $\sum_{n^{\prime}} g_{n^{\prime}} \varphi\left(P_{n^{\prime}}^{\prime}\right) \leq \sum_{n} g_{n} \varphi\left(P_{n}\right)$, where $P_{n^{\prime}}^{\prime}\left(P_{n}\right)$ is the $n^{\prime}$ th (nth) row of $P^{\prime}(P)$.

Where $\left(P_{f}, g_{f}\right)$ and $\left(P_{f}^{\prime}, g_{f}^{\prime}\right)$ are Bayesian derivations from fundamentals $(f, \Pi)$ and $\left(f, \Pi^{\prime}\right)$, respectively, it is the case that if $\left(P_{f}^{\prime}, g_{f}^{\prime}\right)$ is ordered ahead of $\left(P_{f}, g_{f}\right)$ by Blackwell's criterion for some $f$, then it is so ordered for all $f$. This is convenient in that the ordering is then well defined on the original structures $(Y, \Pi)$ and $\left(Y^{\prime}, \Pi^{\prime}\right)$.

The characterization of Blackwell's criterion in Theorem 1 lends itself well to interpretation, toward which we offer the following conceptualization. Suppose that, rather 
than being a signal containing information about a realized state, each element $y_{n^{\prime}}^{\prime} \in Y^{\prime}$ represents a stochastic process that generates the final state, where row $n^{\prime}$ of matrix $P^{\prime}$ represents the distribution over states effected by the $n^{\prime}$ th process. The elements of $Y$ are themselves stochastic processes over the elements of $Y^{\prime}$, where $T_{n n^{\prime}}$ is the probability that the $n^{\prime}$ th process in $Y^{\prime}$ is the one that generates $\omega$ given that $y_{n}$ is realized. Which element $y_{n} \in Y$ obtains is generated according to the probability vector $g$. Under this structure, it is evident that the interim distribution over states given that element $y_{n}$ has been realized is $\sum_{n^{\prime}} T_{n n^{\prime}} P_{n^{\prime}}^{\prime}$, or $P_{n}$ under the original assumptions. Furthermore, the ex-ante probability that element $y_{n^{\prime}}^{\prime}$ generates $\omega$ is $\sum_{n} g_{n} T_{n n^{\prime}}$, which fulfills the second condition of the hypothesis, that $g^{\prime}=g T$. Thus, this conceptualization, in which the fundamentals are $g, P^{\prime}$ and $T$, reproduces all of the relevant features of the interpretation of the elements of $Y$ and $Y^{\prime}$ as signals, in which the fundamentals are $g, P, g^{\prime}$ and $P^{\prime}$, with $P=T P^{\prime}$ and $g^{\prime}=g T$.

Under the above conceptualization, we can interpret observation of an element $y$ in $Y$ as occurring in advance of the resolution of noise as represented by $T$, and observation of an element $y^{\prime}$ in $Y^{\prime}$ as revealing the resolution of that noise. Specifically, suppose that a realized $y$ is revealed, followed at a later point in time by a realized $y^{\prime}$ and, later yet, a realized $\omega$. Consider now an economy that can trade after the observation of a realized $y$ in $Y$. This economy is able to condition allocations on the realization of $y^{\prime}$, as well as on the realization of $\omega$. Would this economy wish to condition its allocation on the realization of $y^{\prime}$ ? The intuition of optimization under risk aversion tells us that it is in fact desirable for such an allocation not to depend on $y^{\prime}$, because such dependence effectively exposes risk-averse consumers to the state-independent noise inherent in $T$. That is, consumers value not only the opportunity to insure against risk generated by variation in the realized state of nature, but also the opportunity to insure against variation in posterior beliefs about the final state.

This intuition is formalized in the first of our main results relating Blackwell's ordering to the value of public information:

Theorem 3: For any two information structures $\zeta$ and $\zeta^{\prime}$, if $\zeta^{\prime}$ is ordered ahead of $\zeta$ by Blackwell's criterion, then $\left(\zeta, \zeta^{\prime}\right) \in S^{\Delta}$. 
Proof: See appendix.

We provide an outline of the proof here. The proof is executed by choosing an arbitrary implementable lottery given $\zeta^{\prime},\left(X_{\Delta}, \delta^{\prime}\right)$, and constructing a lottery given $\zeta$ that is implementable and duplicates the distribution over state-specific allocations of $\left(X_{\Delta}, \delta^{\prime}\right)$. Because $\zeta^{\prime}$ is ordered ahead of $\zeta$ by Blackwell's criterion, there exists an $N \times N^{\prime}$ Markov matrix $T$ such that $P=T P^{\prime}$ and $g^{\prime}=g T$. The probability the candidate lottery under $\zeta$ puts on allocation $j$ in state $\omega_{m}$ given signal $y_{n} \in Y$ is a weighted average of the the probabilities put on $j$ in $\omega_{m}$ given the signals $y_{n^{\prime}}^{\prime} \in Y^{\prime}$, where the weights are given by $T_{n n^{\prime}} P_{n^{\prime} m}^{\prime} / P_{n m}$. In essence, the distribution $\delta$ chooses an element of $y^{\prime} \in Y^{\prime}$ by simulating the noise described by matrix $T$, then uses the probabilities $\delta_{j}^{\prime}\left(y^{\prime}, \omega\right)$. This duplicates the conditional distribution over $X_{\Delta}$ given any state of $\delta^{\prime}$; it is individually rational because by individual rationality of $\left(X_{\Delta}, \delta^{\prime}\right)$, individual rationality is satisfied for all realizations of the simulated noise.

Proposition 1 and Theorem 3 taken together clearly imply that if $\zeta^{\prime}$ is ordered ahead of $\zeta$ by Blackwell's criterion, then $\left(\zeta, \zeta^{\prime}\right) \in S$, and thus independent proof is not required. However, a direct proof is transparent using the intuition of Theorem 3. There, it is shown that, under information structure $\zeta$, it is possible to duplicate the distribution over final allocations of any implementable lottery under $\zeta^{\prime}$ by artificially choosing a signal $y_{n^{\prime}}$ via the probability distribution $T_{n n^{\prime}} P_{n^{\prime} m}^{\prime} / P_{n m}$ when signal $y_{n}$ is observed, and then replicating the $y_{n^{\prime}}$-contingent lottery. Now, suppose that the implementable lottery under $\zeta^{\prime}$ in question is degenerate, with associated allocation $\left(x_{i}\left(y_{n^{\prime}}^{\prime}, \omega_{m}\right)\right)_{i=1}^{I}$. Since a lottery under $\zeta$ that chooses allocation $\left(x_{i}\left(y_{n^{\prime}}^{\prime}, \omega_{m}\right)\right)_{i=1}^{I}$ with probability $T_{n n^{\prime}} P_{n^{\prime} m}^{\prime} / P_{n m}$ when $y_{n}$ is observed yields all consumers an identical ex-ante payoff, the degenerate allocation in which when signal $y_{n}$ and state $\omega_{m}$ are realized the consumers receive the allocation

$$
\left(\sum_{n^{\prime}} \frac{T_{n n^{\prime}} P_{n^{\prime} m}^{\prime}}{P_{n m}} x_{i}\left(y_{n^{\prime}}^{\prime}, \omega_{m}\right)\right)_{i=1}^{I}
$$

necessarily yields them a greater payoff, as they are risk averse. This allocation effectively insures consumers against the resolution of the noise of $T$ by giving them their expected $y_{n^{\prime}}^{\prime}$ allocation given $y_{n}$ in every state, thus formalizing the intuition provided previous to the statement of Theorem 3. 
Because there has been a past emphasis on competitive equilibrium outcomes, one may argue that the set of implementable allocations as we have defined it is too broad. In particular, since competitive equilibrium outcomes are interim Pareto efficient under weak conditions, it may be preferable to limit attention to the subset of implementable allocations that are interim Pareto efficient given the information structure that obtains. This would be appropriate if the nature of implementation were, for instance, that a social planner effects a temporary allocation $\left(x_{i}(y, \omega)\right)_{i=1}^{I}$, but consumers are allowed to trade starting from this temporary allocation before the state $\omega$ is realized. Then only interim Pareto efficient allocations by the planner will be immune to further trade by consumers.

Under conditions for which the interim Pareto frontier is well-defined for all signals, it is easy to establish that the implications of our result also hold when the set of allocations considered are implementable and interim Pareto efficient. The interim Pareto frontier is well-defined if for any signal $y$, there is a partition of the set of implementable allocations $\{A(y), B(y)\}$ with the property that every allocation in $A(y)$ is interim Pareto efficient, and every allocation in $B(y)$ is interim weakly Pareto dominated by some allocation in $A(y) ; A(y)$ is then the interim Pareto frontier for signal $y$.

Theorem 4: For any economy $E \in \mathcal{E}$ such that interim Pareto frontiers are welldefined, if information structure $\zeta^{\prime}$ is ordered ahead of $\zeta$ by Blackwell's criterion, then any interim Pareto efficient implementable allocation in $X^{*}\left(E, \zeta^{\prime}\right)$ is ex-ante weakly Pareto dominated by some interim Pareto efficient allocation in $X^{*}(E, \zeta)$.

Proof: By Theorem 3, any interim Pareto efficient allocation in $X^{*}\left(E, \zeta^{\prime}\right)$ is ex-ante weakly Pareto dominated by some allocation in $X^{*}(E, \zeta)$. For well-defined interim Pareto frontiers, any allocation in $X^{*}(E, \zeta)$ is ex-ante weakly Pareto dominated by some interim Pareto efficient allocation in $X^{*}(E, \zeta)$. Ex-ante weak Pareto dominance is a transitive ordering.

Having established that our public information orderings reverse those of Blackwell, the second issue is whether the obverse is true, so that the set of ordered pairs of information structures that we define and the set that Blackwell defines are in fact mirror images of each other. In other words, if information structure $\zeta$ is better than $\zeta^{\prime}$ in our sense, is 
$\zeta$ necessarily worse than $\zeta^{\prime}$ in Blackwell's sense? Our second main result is that this is indeed the case:

Theorem 5: For any two information structures $\zeta$ and $\zeta^{\prime}$, if $\left(\zeta, \zeta^{\prime}\right) \in S$, then $\zeta^{\prime}$ is ordered ahead of $\zeta$ by Blackwell's criterion.

Proof: See appendix.

The proof establishes the contrapositive of the proposition by construction: if $\zeta^{\prime}$ is not ordered ahead of $\zeta$ by Blackwell's criterion, then an economy $\hat{E}$ can be found such that $\left(\zeta, \zeta^{\prime}\right) \notin S(\hat{E})$. Note that Theorem 5 also confirms the equivalence of sets $S$ and $S^{\Delta}$.

\section{DISCUSSION}

While the intuition provided by the conceptualization of signal posteriors as processes generating the final state makes the forces behind our results fairly clear, we offer here some additional analysis. The exact inverse relationship between the value of information to an individual decision-maker and the value of public information to an economy under risk strongly suggests some formal duality between the two. This duality is most easily understood via the characterization of Blackwell's ordering of Theorem 2.

Consider two decision problems. The first is the standard one of Blackwell: there is a single decision maker who chooses an action $a \in A$, where $A$ is some compact set, and has von Neumann-Morgenstern utility $u(a, \omega)$. The decision maker is allowed to observe the realization of a signal $y_{n}$ before choosing contingent action $a_{n}$. Thus, for any signal $y_{n}$, she will choose $a_{n} \in \operatorname{argmax}_{a \in A} \sum_{m} P_{n m} u\left(a, \omega_{m}\right)$, and her ex-ante expected payoff from doing so is

$$
\sum_{n} g_{n} \max _{a_{n} \in A} \sum_{m} P_{n m} u\left(a_{n}, \omega_{m}\right) .
$$

The function $\max _{a_{n} \in A} \sum_{m} P_{n m} u\left(a_{n}, \omega_{m}\right)$ is necessarily convex in $M$-vector $P_{n}$, as it is linear in $P_{n}$ for any fixed action $a_{n}$. Thus, if $\left(P^{\prime}, g^{\prime}\right)$ yields a lower expectation of all concave functions $\varphi(\cdot)$ defined on Markov $M$-vectors than does $(P, g)$, it must yield a higher expectation of all convex functions $\varphi^{\prime}(\cdot)$ defined on Markov $M$-vectors than does $(P, g)$, and the decision maker must prefer $\left(P^{\prime}, g^{\prime}\right)$ to $(P, g)$.

Consider now a problem in which the decision maker is a social planner for an economy under risk. The decision maker's problem is to choose a state- and signal-dependent 
allocation for the economy $\left(x_{i}\left(y_{n}, \omega_{m}\right)\right)_{i=1}^{I}$. The allocation she chooses must be implementable under our definition. Her objective function is a standard weighted social welfare function $\sum_{i} \lambda_{i} \sum_{n} g_{n} \sum_{m} P_{n m} u_{i}\left(x_{i}\left(y_{n}, \omega_{m}\right), \omega_{m}\right)$, with $\lambda_{i} \geq 0$ for all $i$. Without the individual rationality constraints, the optimal allocation would simply maximize the social welfare function state-by-state, and would therefore be independent of the realized $y_{n}$. Hence the maximized value of the function for a given $n$ would be linear in $P_{n}$, and in particular would be equivalent for all $g$ and $P$ satisfying $g P=f$. Because the $P_{n}$ enter the individual rationality constraints linearly, the constrained program is thus concave in $P_{n}$ for each realized signal $n$. Thus, the social planner can always attain at least as great a weighted social welfare with less information as she can with more information.

We note a technical similarity between this latter result and that of Schlee [9]. The environments Schlee identifies share the feature that the equilibrium price of a given good in a given state is linear in the assessed probability of that state occurring. Since consumers' signal-dependent indirect utilities are concave in prices, given the linear relationship between posteriors and prices, the indirect utilities are also necessarily concave in the posteriors, and hence consumers achieve worse ex-ante indirect utility when there is more information. Thus, public information will have an unambiguously negative value under competitive equilibrium in any environment for which this linearity between posteriors and equilibrium prices holds.

One natural question in light of the results of this and other papers is whether the negative value of information is preserved under the solution concept of the core. The core properly lies "between" competitive equilibrium and implementable allocations, as any competitive equilibrium allocation is in the core, and any core allocation is implementable. An immediate indication that the core does not preserve the negative value of information is derived from core convergence under replication: the negative value of information is not globally preserved under competitive equilibrium; hence there necessarily exist an economy and two information structures such that the negative value of information is violated for the competitive equilibrium allocations; with sufficient replication of the economy the core converges to the competitive equilibrium allocations; ergo, the negative value of information is also violated under the core. 
A direct proof of the failure of the core to preserve a strictly negative value of information is also easy to construct. Suppose an exchange economy with three consumers and two states of the world. In each state there are two commodities, $x$ and $y$. All consumers have state-independent preferences. Consumer 1 values only commodity $x$, with utility $u_{1}\left(x_{1}, y_{1}\right)=x_{1}$. Consumers 2 and 3 have identical utility functions $u_{i}\left(x_{i}, y_{i}\right)=\sqrt{x_{i}}+y_{i}$, $i \in\{2,3\}$. Consumer 1 is endowed with one unit of good $y$ in both states. Consumer 2 is endowed with one unit of good $x$ in state 1 , nothing in state 2 ; consumer 3 is endowed with one unit of good $x$ in state 2 , nothing in state 1 . Under a signal structure that fully reveals the final state, the allocation in which consumer 1 consumes one unit of good $x$ in both states, consumer 2 consumes one unit of good $y$ in state 1 , and consumer 3 consumes one unit of good $y$ in state 2 , is in the interim core; furthermore, no other feasible state-dependent allocation gives consumer 1 a greater ex-ante utility. Next, consider a signal structure in which no information is revealed, and the ex-ante probability of each of the two states is $1 / 2$. With no aggregate risk, consumers 2 and 3 must be fully insured in any core allocation. Furthermore, the set of utility pairs $\left(u_{2}, u_{3}\right)$ on the Pareto frontier for the coalition of consumers 2 and 3 is $(\sqrt{\alpha}, \sqrt{1-\alpha})$, for $\alpha \in[1 / 4,3 / 4]$. To maximize consumer 1's utility subject to the constraint that the utilities of the consumers 2 and 3 equal a given point on their coalitional Pareto frontier, it is evident that consumer 1 should trade good $y$ to the others so that the others each are left with the same amount of good $x$ (so that their marginal rates of substitution are identical); doing so yields consumer 1 a total of

$$
1-\frac{1}{2}(\sqrt{\alpha}+\sqrt{1-\alpha}-1)
$$

units of $\operatorname{good} x$ in each state. This is strictly less than 1 for all $\alpha \in(0,1)$, and in particular for all $\alpha \in[1 / 4,3 / 4]$. Thus, there is no allocation in the interim core under no information that yields consumer 1 an ex-ante utility of 1 , as there is under perfect information, and an unambiguous negative value of information, in the sense of Pareto dominance, fails.

For what economies might public information have positive value? A clear case is that in which information bears on the efficiency of production. Implicit in our specification of an economy is that the set of state-contingent production possibility sets is the Cartesian product $\Pi_{m=1}^{M} X\left(\omega_{m}\right)$, and hence a rectangle. This specifically rules out cases in which 
the choice of production in one state affects what is feasible in another. However, if important production decisions must be made in advance of the resolution of uncertainty, then such cases may apply. An obvious example is sunk investment in a specific uncertain technology; the resources that are sunk could be put to different uses, and hence the decision to invest affects the production possibilities set in all states. In that environment, improved information about the technology would allow a welfare-improving adjustment in the amount of investment. So for instance, in a Robinson Crusoe economy in which production had this dependency property, more information in Blackwell's sense would always have positive value. In general, if production has this feature then there will be tradeoffs between more efficient production and more efficient risk-sharing, as in Eckwert and Zilcha (2001) in a somewhat different context.

\section{CONCLUSION}

We have attempted to show that the notion raised in Hirshleifer's early paper is quite general: more public information available before trade results in a worse set of attainable outcomes for an economy under uncertainty. Adherence to competitive equilibrium as a solution concept obscures this relationship, as competitive equilibrium may induce comparative statics that are counterintuitive in light of different solution concepts. In this regard, one may interpret the conditions that Schlee [9] identifies as sufficient to guarantee a negative value of public information, as being conditions that induce a natural regularity in comparative statics under competitive equilibrium.

The preciseness of the relationship between Blackwell's ordering of information and the value of public information in an economy under uncertainty may indicate that additional equivalences between Blackwell and the value of information in broad classes of economic environment, such as principal-agent settings, may hold. Any such results would help unify the measurement of information and support Blackwell's ordering as the standard.

\section{APPENDIX}

Proof of Theorem 3. Since Blackwell's criterion is satisfied, there exists an $N \times N^{\prime}$ Markov matrix $T$ such that $P=T P^{\prime}$ and $g T=g^{\prime}$. Fix an arbitrary economy $E \in \mathcal{E}$, and 
let $x_{\Delta}^{\prime} \equiv\left(X_{\Delta}, \delta^{\prime}\right)$ be an arbitrary finite lottery over allocations that is implementable given $E$ and $\zeta^{\prime}$. Consider the lottery $x_{\Delta}^{*} \equiv\left(X_{\Delta}, \delta^{*}\right)$ given $E$ and $\zeta$, satisfying

$$
\delta_{j}^{*}\left(y_{n}, \omega_{m}\right)=\sum_{n^{\prime}=1}^{N^{\prime}} \frac{T_{n n^{\prime}} P_{n^{\prime} m}}{P_{n m}} \delta_{j}^{\prime}\left(y_{n^{\prime}}^{\prime}, \omega_{m}\right)
$$

if $P_{n m}>0,\left(\delta_{j}^{*}\left(y_{n}, \omega_{m}\right)\right)_{j=1}^{J}$ an arbitrary probability vector if $P_{n m}=0 . \delta_{j}^{*}\left(y_{n}, \omega_{m}\right)$ is a proper lottery because $\sum_{j} \delta_{j}^{\prime}\left(y_{n^{\prime}}^{\prime}, \omega_{m}\right)=1$ for all $y_{n^{\prime}}^{\prime}$ and $\omega_{m}$, and $\sum_{n^{\prime}=1}^{N^{\prime}} T_{n n^{\prime}} P_{n^{\prime} m}=$ $P_{n m}$ for all $y_{n}$ and $\omega_{m}$. We will show that this lottery yields an identical distribution over all state-specific allocations and is implementable given $E$ and $\zeta$.

First, we prove that the lotteries are ex-ante identical. Fix allocation $j \in X_{\Delta}$ and state $\omega \in \Omega$. The conditional probability that $j$ is chosen under $x_{\Delta}^{*}$ in state $\omega_{m}$ is

$$
\begin{aligned}
& \sum_{n=1}^{N} \pi\left(y_{n} \mid \omega_{m}\right) \delta_{j}^{*}\left(y_{n}, \omega_{m}\right) \\
= & \sum_{n=1}^{N} \pi\left(y_{n} \mid \omega_{m}\right) \sum_{n^{\prime}=1}^{N^{\prime}} \frac{T_{n n^{\prime}} P_{n^{\prime} m}^{\prime}}{P_{n m}} \delta_{j}^{\prime}\left(y_{n^{\prime}}^{\prime}, \omega_{m}\right) \\
= & \sum_{n=1}^{N} \frac{g_{n}}{f_{m}} \sum_{n^{\prime}=1}^{N^{\prime}} T_{n n^{\prime}} P_{n^{\prime} m}^{\prime} \delta_{j}^{\prime}\left(y_{n^{\prime}}^{\prime}, \omega_{m}\right) \\
= & \sum_{n^{\prime}=1}^{N^{\prime}} \frac{P_{n^{\prime} m}^{\prime}}{f_{m}} \delta_{j}^{\prime}\left(y_{n^{\prime}}^{\prime}, \omega_{m}\right) \sum_{n=1}^{N} T_{n n^{\prime}} g_{n} \\
= & \sum_{n^{\prime}=1}^{N^{\prime}} \frac{g_{n^{\prime}} P_{n^{\prime} m}^{\prime}}{f_{m}} \delta_{j}^{\prime}\left(y_{n^{\prime}}^{\prime}, \omega_{m}\right) \\
= & \sum_{n^{\prime}=1}^{N^{\prime}} \pi^{\prime}\left(y_{n^{\prime}}^{\prime} \mid \omega_{m}\right) \delta_{j}^{\prime}\left(y_{n^{\prime}}^{\prime}, \omega_{m}\right) .
\end{aligned}
$$

The last term is the conditional probability that $j$ is chosen under $x_{\Delta}^{\prime}$ in state $\omega_{m}$.

Because the ex-ante distributions over state-specific allocations are identical, we have that for any $j, y \in Y$ and $\omega \in \Omega$, if $\delta_{j}(y, \omega)>0$ then $\delta_{j}^{\prime}\left(y^{\prime}, \omega\right)>0$ for some $y^{\prime}$. Thus, feasibility of $\left(X_{\Delta}, \delta^{\prime}\right)$ implies feasibility of $\left(X_{\Delta}, \delta\right)$. Individual rationality is satisfied if for all $y_{n} \in Y$ and all consumers $i$,

$$
g_{n} \sum_{m=1}^{M} P_{n m} \sum_{j} \delta_{j}^{*}\left(y_{n}, \omega_{m}\right)\left(u_{i}\left(x_{i j}, \omega_{m}\right)-u_{i}\left(e_{i}\left(\omega_{m}\right), \omega_{m}\right)\right) \geq 0 .
$$


Clearly, the specification of $\delta_{j}^{*}\left(y_{n}, \omega_{m}\right)$ does not matter if $g_{n}=0$, or if $P_{n m}=0$. Otherwise, substituting the proposed $\delta_{j}^{*}\left(y_{n}, \omega_{m}\right)$ we have

$$
\begin{aligned}
& g_{n} \sum_{m=1}^{M} P_{n m} \sum_{j} \sum_{n^{\prime}=1}^{N^{\prime}} \frac{T_{n n^{\prime}} P_{n^{\prime} m}}{P_{n m}} \delta_{j}^{\prime}\left(y_{n^{\prime}}^{\prime}, \omega_{m}\right)\left(u_{i}\left(x_{i j}, \omega_{m}\right)-u_{i}\left(e_{i}\left(\omega_{m}\right), \omega_{m}\right)\right) \\
= & g_{n} \sum_{n^{\prime}=1}^{N^{\prime}} T_{n n^{\prime}} \sum_{m=1}^{M} P_{n^{\prime} m} \sum_{j} \delta_{j}^{\prime}\left(y_{n^{\prime}}^{\prime}, \omega_{m}\right)\left(u_{i}\left(x_{i j}, \omega_{m}\right)-u_{i}\left(e_{i}\left(\omega_{m}\right), \omega_{m}\right)\right) .
\end{aligned}
$$

Because $T$ is nonnegative and $x_{\Delta}^{\prime}$ is individually rational by hypothesis, each of the $N^{\prime}$ terms in the sum across $n^{\prime}$ is nonnegative, so the entire sum is nonnegative and $x_{\Delta}^{*}$ is individually rational.

Proof of Theorem 5. We prove the contrapositive using the characterization of Blackwell's criterion in Theorem 2. Specifically, we show that if the conditions of Theorem 2 do not hold, then we can construct an economy $E \in \mathcal{E}$ and find an allocation in $X^{*}\left(E, \gamma^{\prime}\right)$ that is not weakly Pareto dominated by any allocation in $X^{*}(E, \zeta)$, implying that $\left(\zeta, \zeta^{\prime}\right) \notin S$. Thus, we take as a hypothesis that there exists a concave function $\varphi(\cdot)$ defined on Markov $M$-vectors such that $\sum_{n^{\prime}} g_{n^{\prime}} \varphi\left(P_{n^{\prime}}^{\prime}\right)>\sum_{n} g_{n} \varphi\left(P_{n}\right)$. If such a $\varphi(\cdot)$ exists, then there is necessarily a concave function $\varphi^{\prime}(\cdot)$ with the same property that takes on only nonnegative values (e.g., via an affine transformation of $\varphi(\cdot)$ if $\varphi(\cdot)$ is finite-valued, or via an adjustment in $\varphi(\cdot)$ so that it is finite-valued but preserves the inequality, followed by an affine transformation). Thus, we assume without loss of generality that $\varphi(\cdot)$ is nonnegative-valued.

The economy $E$ an exchange economy of $M+1$ consumers and $2 M$ discrete goods in each state. A quantity of good $m$ in state $m^{\prime}$ will be denoted $x_{m^{\prime}}^{m}$. For $m \leq M$, consumer $m$ has von Neumann-Morgenstern utility $x^{m}+x^{M+m}$ in every state. That is, each of the first $m$ consumers is risk-neutral, and cares only about two goods, the $m$ th and $M+m$ th. Consumer $m \leq M$ is endowed with one unit of good $m$ in every state, i.e., $e_{m}^{m}\left(\omega_{m^{\prime}}\right)=1$ for all $m^{\prime} \in\{1, \ldots, M\}$.

Consumer $M+1$ also has state-independent utility, and cares only about goods $m \leq M$, so her von Neumann-Morgenstern utility function can be defined solely on nonnegative $M$-vectors. This utility function, called $u(\cdot)$, is constructed from function $\varphi(\cdot)$ as follows: 
$u(0)=0$, and for any nonzero, nonnegative bundle of the first $M$ goods $\left\{x^{1}, x^{2}, \ldots, x^{M}\right\}$, we have

$$
u\left(x^{1}, \ldots, x^{M}\right)=\left(\sum_{m=1}^{m} x^{m}\right) \varphi\left(\frac{x^{1}}{\sum_{m=1}^{m} x^{m}}, \ldots, \frac{x^{M}}{\sum_{m=1}^{m} x^{m}}\right) .
$$

$u(\cdot)$ has the property that it coincides with $\varphi(\cdot)$ on all Markov $M$-vectors. Because $\varphi(\cdot)$ is concave by hypothesis, $u(\cdot)$ is as well and hence is permissible under the restrictions on economies in $E$. Consumer $M+1$ is endowed with 1 unit of good $M+m$ in state $m$ for all $m \leq M$, i.e., $e_{M+1}^{M+m}\left(\omega_{m}\right)=1$ for all $m \in\{1, \ldots, M\}$.

This completes the description of the economy. We now focus on a particular allocation under information structure $\zeta^{\prime}$. In this allocation, for any consumer $m \leq M$, we have $x^{m}\left(y_{n^{\prime}}^{\prime}, \omega_{m^{\prime}}\right)=\left(1-P_{n^{\prime} m}\right)$ for all $n^{\prime}$ and $m^{\prime} \in\{1, \ldots, M\}$, and $x^{M+m}\left(y_{n^{\prime}}^{\prime}, \omega_{m}\right)=1$ for all $n^{\prime}$. That is, given realized signal $n^{\prime}$, consumer $m$ is allocated $1-P_{n^{\prime} m}^{\prime}$ units of good $m$ in all states, and 1 unit of good $M+m$ in state $m$. For consumer $M+1$ and every good $m \leq M$, we have $x^{m}\left(y_{n^{\prime}}^{\prime}, \omega_{m^{\prime}}\right)=P_{n^{\prime} m}$ for all $n^{\prime}$ and $m^{\prime} \in\{1, \ldots, M\}$, i.e., for any good $m \leq M$ and signal $n^{\prime}$, consumer $M+1$ is allocated $P_{n^{\prime} m}^{\prime}$ units in all states. This allocation is feasible, and because it gives all consumers $m \leq M$ their endowment utility of 1 , while giving consumer $M+1$ strictly positive utility rather than her endowment utility of 0 , it is individually rational, and hence implementable.

To complete the proof, we show that the above allocation is not weakly Paretodominated by any allocation that is implementable under $\zeta$. First, note that because consumer $M+1$ is interim perfectly insured and receives nonnegative good quantities summing to 1 in all states, her interim expected utility given signal $n^{\prime}$ under this allocation is simply $\varphi\left(P_{n^{\prime}}^{\prime}\right)$, and her ex-ante expected utility is exactly $\sum_{n^{\prime}} g_{n^{\prime}} \varphi\left(P_{n^{\prime}}^{\prime}\right)$. Furthermore, this is the allocation that maximizes consumer $M+1$ 's utility subject to the constraint that all other consumers maintain their endowment utility. To see this, note that the only Pareto-improving reallocations from the endowment that are possible in this economy entail consumer $M+1$ trading all of her good $M+m$ in state $m$ (which she does not value) to consumer $m$ (the only consumer who values good $M+m$ ) for a quantity of good $m$ (the only good consumer $m$ has to trade). Because consumer $M+1$ is risk-averse, it is efficient for her to consume the same amount of good $m$ in all states. The largest amount of good $m$ in all states that consumer $m$ would be willing to trade for 1 unit of good $M+m$ in state $m$ (which he values at $P_{n^{\prime} m}^{\prime}$ utils) is $P_{n^{\prime} m}^{\prime}$. 
Thus, there is no different allocation that yields a greater interim utility for consumer $M+1$ and respects individual rationality for the remaining consumers. This means that

$\sum_{n^{\prime}} g_{n^{\prime}} \varphi\left(P_{n^{\prime}}^{\prime}\right)$ is the largest possible ex-ante expected utility consumer $M+1$ can attain among all implementable allocations under $\zeta^{\prime}$. However, the same argument establishes that $\sum_{n} g_{n} \varphi\left(P_{n}\right)$ is the largest utility consumer $M+1$ can attain among all implementable allocations under $\zeta$. As $\sum_{n^{\prime}} g_{n^{\prime}} \varphi\left(P_{n^{\prime}}^{\prime}\right)>\sum_{n} g_{n} \varphi\left(P_{n}\right)$ by hypothesis, there is no allocation implementable under $\zeta$ yielding consumer $M+1$ as great an ex-ante expected utility as the constructed allocation under $\zeta^{\prime}$, and hence no allocation implementable under $\zeta$ that weakly ex-ante Pareto dominates the constructed allocation, and thus $\left(\zeta, \zeta^{\prime}\right) \notin S$.

\section{REFERENCES}

1. D. Blackwell, "Comparison of Experiments," in J. Neymann, ed., Proceedings of the Second Berkeley Symposium on Mathematical Statistics and Probability. Berkeley: University of California Press, 1951, 93-102.

2. D. Blackwell and M. A. Girshick Theory of Games and Statistical Decisions. New York: John Wiley and Sons, 1954.

3. B. EckWert and I. Zilcha, Incomplete Risk-Sharing Arrangements and the Value of Information, Journal of Economic Theory 100 (2001), 172-186.

4. J. Green, Value of Information with Sequential Futures Markets, Econometrica 49 (1981), 335-358.

5. J. Hakansson, G. Kunkel and J. A. Ohlson, Sufficient and Necessary Conditions for Information to have Social Value in Pure Exchange, Journal of Finance 37 (1982), 1169-1181.

6. J. Hirshleifer, The Private and Social Value of Information and the Reward to Incentive Activity, American Economic Review 61 (1971), 561-574.

7. R. E. Kinlstrom, "A 'Bayesian' Exposition of Blackwell's Theorem on the Comparison of Experiments," in M. Boyer and R. E. Kihlstrom, eds., Bayesian Models of Economic Theory. Amsterdam: North-Holland, 1984.

8. J. M. Marshall, Private Incentives and Public Information, American Economic Review 64 (1974), 373-390. 
9. E. E. SchleE, The Value of Information in Efficient Risk Sharing Arrangements, American Economic Review 91 (2001), 509-524. 